\title{
NONTHERMAL ELECTRON BERNSTEIN EMISSION IN NSTX-LIKE DISCHARGES
}

\author{
R. W. HARVEY,* A. P. SMIRNOV, and E. NELSON-MELBY CompX, Del Mar, California \\ G. TAYLOR Princeton Plasma Physics Laboratory, New Jersey
}

S. CODA Ecole Polytechnique Federale de Lausanne, Lausanne, Switzerland

A. K. RAM Massachusetts Institute of Technology, Boston, Massachusetts

Received March 1, 2007

Accepted for Publication June 18, 2007

In overdense plasma for which the plasma frequency exceeds the cyclotron frequency, X-mode, nearperpendicular cyclotron emission does not propagate to the outboard plasma edge. However, under these conditions it remains possible for electron Bernstein waves $(E B W s)$ to transmit emitted radiation from central plasma to the plasma exterior via a mode conversion to electromagnetic waves near the plasma edge. GENRAY is an all-frequencies, three-dimensional ray-tracing code and also calculates EBW emission (EBWE) from thermal or nonthermal relativistic distributions. The numerical methods are based on the earlier HORACE circular plasma code (R.W. Harvey et al., Proc. 7th Joint Workshop and International Atomic Energy Agency Technical Committee Meeting on Electron Cyclotron Emission and Electron Cyclotron Resonance Heating, Hefei, China, 1989), generalized to noncircular plasmas and to elec- tromagnetic EBWs, including a parallel refractive index greater than 1. Emission and absorption are calculated on an array of points along EBW rays emanating from the antenna, and the radiation transport equation is backsolved along the EBW rays to the antenna. Hot plasma dispersion is used along with a relativistic calculation of the thermal or nonthermal emission and absorption. This paper describes the calculation and reports new results for nonthermal EBWE. Along with detailed numerical analysis, EBWE can be used to measure both thermal and nonthermal properties of the electron distribution function.

KEYWORDS: plasmas, radio-frequency heating, electron Bernstein wave emission

Note: Some figures in this paper are in color only in the electronic version.

\section{INTRODUCTION}

An examination of the Clemmow-Mullaly-Allis diagram $^{1}$ for cold plasma waves reveals that in overdense plasma such that $\omega_{p e} / \omega_{c e}$ is $>1$, the usual X-mode cyclotron radiation cannot propagate from the plasma interior to the plasma edge; instead, the emitted energy will be intercepted by the upper hybrid resonance (UHR). However, under overdense conditions hot plasma electron Bernstein waves (EBWs) can propagate from the plasma core to near the plasma edge where they are mode converted to electromagnetic waves and continue to the antenna in the exterior, vacuum region.

\footnotetext{
*E-mail: bobh@compxco.com
}

EBW emission (EBWE) is studied for two reasons: first, as a diagnostic in its own right for plasma radiation temperature ${ }^{2}$ and second, in support of future EBW current drive experiments in high-beta, overdense plasma such as can be obtained in the National Spherical Torus Experiment $^{3}$ (NSTX), Mega Ampère Spherical Toka$\mathrm{mak}^{4}$ (MAST), and PEGASUS ${ }^{5}$ spherical plasma, and in the Madison Symmetric Torus ${ }^{5}$ (MST) reversed field plasma devices. By reciprocity, it is expected that if plasma conditions are such that there is efficient coupling of EBWE out of the plasma, then the ray path can be reversed, and good coupling of EBW for heating and current drive purposes can be obtained. Particularly, for the EBW current drive, it is calculated that substantial nonthermal electron distributions will be formed. ${ }^{3,5}$ For these 
reasons, this paper considers EBWE from thermal and nonthermal electron distributions in the NSTX plasma.

The GENRAY ray-tracing $\operatorname{code}^{6}$ has been augmented to calculate EBWE from general, gyrotropic distributions (and is also an all-frequencies ray-tracing code). Emission and absorption coefficients are calculated at each point along EBW rays originating at the antenna, and the radiation transport equation ${ }^{7}$ for radiation intensity $I$ is backsolved to the antenna, as described in Ref. 8 . The emission ray trajectories are calculated using a hot plasma dispersion relation giving the wave numbers and polarizations, along with a numerical, fully relativistic damping calculation. These ray parameters are used to obtain the coefficients. Within the open literature, this paper contains the first description of the GENRAY emission calculation of integrated radiation to the plasma edge and presents first results (to the authors' knowledge) for integrated EBWE from nonthermal electron distributions.

To bring the radiation out of the plasma as free space electromagnetic modes, there are two possible mode conversion scenarios near the plasma edge, Bernstein-X mode conversion (BX) and Bernstein-X-O mode conversion (BXO), depending on the plasma density gradients and the parallel wave number. ${ }^{9}$ The BX mode conversion is problematic, requiring very strong density gradients. This paper focuses on the less demanding $\mathrm{BXO}$ emission window, which occurs for oblique angles of incidence on the mode conversion layer at wave frequency $\omega=\omega_{p e}$ (Refs. 10, 11, and 12). The appropriate angle of incidence is found with a shooting algorithm to obtain the central ray angles giving $100 \%$ transmission, for a specified receiver (antenna) position. A new feature here for EBWE is that the relativistic resonance condition changes character at parallel refractive index $n_{\|}>1$ from the usual ellipse to a hyperbola. ${ }^{5}$

In the following, we show calculated EBWE radiation temperature profiles for an NSTX experimental profile with thermal distributions and compare them to a case with EBW current drive nonthermal distributions from a simulated Ordinary eXtraordinary Bernstein (OXB) injection experiment. Both "low-" and high-beta cases are considered. EBW waves are slow, near-electrostatic, and strongly damped waves, ${ }^{5}$ like lower hybrid waves, and in contrast to the usual O-mode and X-mode electron cyclotron waves. Depending on $n_{\|}$, absorption and emission may be strong from bulk or tail electrons.

There is major sensitivity of the radiation as to whether the observed frequency is just above or just below a cyclotron harmonic. In the former case the observed radiation comes primarily from near thermal electrons, whereas in the latter case it comes more from the higher-velocity nonthermal electrons. The results are quite dependent on the poloidal angle at which the antenna is located. We find that EBWE provides a flexible means to examine both thermal and nonthermal electron distributions.

\section{GENRAY IMPLEMENTATION OF EMISSION}

\section{II.A. The Radiation Transport Equation}

The GENRAY ray-tracing code calculates emission following the model proposed in Ref. 8. In the WKB approximation, the radiation transport equation ${ }^{7}$ gives radiation intensity $I$, the power in each mode per unit area-radian frequency-steradian flowing toward the antenna along the rays in the plasma:

$$
n_{r}^{2} \hat{s} \cdot \nabla\left(n_{r}^{-2} I\right)=j-\alpha I
$$

where

$$
\begin{aligned}
\hat{s}= & \text { unit vector along the ray trajectory } \\
& \text { for the mode under consideration } \\
n_{r}= & \text { ray refractive index }{ }^{7} \text { emissivity } \\
j= & \text { power radiated by the plasma per } \\
& \text { unit volume-radian frequency- } \\
& \text { steradian } \\
\text { absorptivity } \alpha= & \text { inverse wave energy damping } \\
& \text { length along the ray. }
\end{aligned}
$$

The ray emission trajectories are calculated from the solution of the ray-tracing equations. These trajectories are chosen to terminate at the antenna and by reciprocity are obtained by ray tracing back into the plasma. Various dispersion relations are available in the code: cold, nonrelativistic hot, and fully relativistic plasma. Here, we use the nonrelativistic, hot Maxwellian plasma disper$\operatorname{sion}^{1,5}$ for the ray paths, wave numbers, and electric field polarizations and neglect the nonthermal effects except as they affect the emission and absorption. The nonthermal effects are obtained from fully relativistic absorption and emission coefficients given in Refs. 1, 7, and 13:

$$
\alpha=\frac{\omega}{4 \pi} \frac{\boldsymbol{E}^{*} \cdot \boldsymbol{\varepsilon}_{a} \cdot \boldsymbol{E}}{|\boldsymbol{S}|}
$$

and

$$
j=\pi n_{r}^{2}\left(\frac{\omega}{c}\right)^{2} \frac{\boldsymbol{E}^{*} \cdot \boldsymbol{G} \cdot \boldsymbol{E}}{|\boldsymbol{S}|},
$$

where

$$
\begin{aligned}
\boldsymbol{E}=\boldsymbol{E}(\omega, \boldsymbol{k})= & \text { space-time Fourier transform of the } \\
& \text { electric field } \\
\boldsymbol{S}(\omega, \boldsymbol{k})= & \text { energy flux density per frequency and } \\
& \text { per unit volume of } \boldsymbol{k} \text { space } \\
\boldsymbol{S}= & U \boldsymbol{v}_{g} \\
\boldsymbol{v}_{g} \equiv \partial \omega / \partial \boldsymbol{k}= & \text { wave group velocity. }
\end{aligned}
$$

We use the conventions in Refs. 7 and 13, which give spectral energy density $U=(1 / 8 \pi)\left(\boldsymbol{B}^{*} \cdot \boldsymbol{B}+\boldsymbol{E}^{*} \cdot\left(\partial\left(\omega \boldsymbol{\varepsilon}_{h}\right) /\right.\right.$ $\partial \omega) \cdot \boldsymbol{E})$. In this expression we use Hermitian dielectric 
tensor elements for different dielectric tensors used in ray-trajectory calculations according to the selected dispersion relation. It is essential that a relativistic expression be used for anti-Hermitian dielectric tensor elements $\boldsymbol{\varepsilon}_{\mathrm{a}}$ and the corresponding correlation tensor $\boldsymbol{G}$ for the fluctuating current density. These are obtained locally in space from the momentum relativistic electron distribution function $f$, which is brought into GENRAY from the CQL3D Fokker-Plank code ${ }^{14}$ :

$$
\begin{aligned}
\boldsymbol{\varepsilon}_{a}= & -\pi \frac{\omega_{p}^{2}}{\omega^{2}} \sum_{n=-\infty}^{\infty} \\
& \times \int d^{3} p U(f) S^{(n)} \delta\left(\gamma-\frac{k_{\|} v_{\|}}{\omega}-\frac{n \omega_{c}}{\omega}\right)
\end{aligned}
$$

and

$$
\begin{aligned}
\boldsymbol{G}= & \frac{\pi}{(2 \pi)^{5}} \frac{\omega_{p}^{2}}{\omega^{2}} \frac{1}{m} \sum_{n=-\infty}^{\infty} \\
& \times \int \frac{d^{3} p}{\gamma} f p_{\perp} S^{(n)} \delta\left(\gamma-\frac{k_{\|} v_{\|}}{\omega}-\frac{n \omega_{c}}{\omega}\right),
\end{aligned}
$$

where

$$
\begin{aligned}
U(f) & \equiv \frac{1}{\gamma}\left(\frac{n \omega_{c}}{\omega} \frac{\partial f}{\partial p_{\perp}}+n_{\|} \frac{p_{\|}}{m c} \frac{\partial f}{\partial p_{\|}}\right) \\
S^{(n)} \equiv & {\left[\begin{array}{ccc}
p_{\perp}\left(\frac{n J_{n}}{b}\right)^{2} & i p_{\perp} \frac{n J_{n} J_{n}^{\prime}}{b} & p_{\|} \frac{n J_{n}^{2}}{b} \\
-i p_{\perp} \frac{n J_{n} J_{n}^{\prime}}{b} & p_{\perp}\left(J_{n}^{\prime}\right)^{2} & -i p_{\|} J_{n} J_{n}^{\prime} \\
p_{\|} \frac{n J_{n}^{2}}{b} & i p_{\|} J_{n} J_{n}^{\prime} & \frac{p_{\|}^{2}}{p_{\perp}} J_{n}^{2}
\end{array}\right] } \\
v_{\|} / \gamma m & =\text { parallel velocity } \\
m & =\operatorname{rest~mass} \\
\gamma & =\left(1+p^{2} / m^{2} c^{2}\right)
\end{aligned}
$$

and $f\left(p_{\|}, p_{\perp}\right)$ is normalized to $\int d^{3} p f=1$. The Bessel function $J_{n}$ and its derivative $J_{n}^{\prime}$ are evaluated at argument $b=k_{\perp} p_{\perp} / m \omega_{c}$. The directions $\|$ and $\perp$ are referenced to the ambient magnetic field. Using the constants of motion, the local distribution function $f$ along each ray is obtained from the distributions evaluated at the minimum magnetic field point on a flux surface, for example, by the CQL3D bounce-averaged Fokker-Planck code. ${ }^{14}$ For given $f$, the tensors $\boldsymbol{\epsilon}_{a}$ and $\boldsymbol{G}$ are evaluated with a one-dimensional numerical integration in momentum space along the resonance curve argument of the above delta functions.

We also add a collisional absorption coefficient $\alpha_{\text {collision }}$ into the transport equation

$$
n_{r}^{2} \frac{d}{d s}\left(n_{r}^{-2} I\right)=j-\left(\alpha+\alpha_{\text {collision }}\right) I,
$$

where

$$
\begin{aligned}
\alpha_{\text {collision }} & =\frac{2 v_{e i}}{v_{g}} \\
v_{e i} & =\text { electron-ion collision frequency } \\
v_{t} & =\text { group velocity. }
\end{aligned}
$$

The collisional absorption can be essential at the plasma edge if the plasma temperature is low but the plasma density is moderate. However, in GENRAY we ignore the additional emission because of the collisions in the low-temperature edge plasma, considering only the absorption of outgoing radiation coming from the highertemperature regions.

\section{II.B. Radiation Temperature}

The emission intensity at the antenna is obtained by integration over ray length $s$ :

$$
I_{0}(s)=n_{r}^{2}(0) \int_{0}^{s} \frac{j(t)}{n_{r}^{2}(t)} d t \exp \left(-\int_{0}^{t} \alpha(q) d q\right)
$$

where $s$ is length along the ray trajectory starting just inside the plasma edge, where $s=0$. The ray radiation temperature can be calculated using the total radiation $I_{0}(L)$ at the plasma edge ${ }^{7}$ :

$$
T_{\text {rad }}=\frac{2 \pi c^{2} I_{0}(L)}{f^{2}} .
$$

It is convenient to introduce the specific intensity,

$$
I_{0}^{\prime}(s)=\frac{d I_{0}(s)}{d s}=n_{r}^{2}(0) \frac{j(s)}{n_{r}^{2}(s)} \exp \left(-\int_{0}^{s} \alpha(q) d q\right),
$$

which gives the emission $d I_{0}=I_{0}^{\prime}(s) d s$ at the plasma edge, i.e., to the antenna, originating from the ray bin $(s, s+d s)$. This function clarifies the spatial region responsible for the emission observed at the detector. The main contribution to the total radiation $I_{0}(L)$ is generally from ray bins near the point where specific intensity has maximal value. The absorption and emission coefficients form peaks along the ray, as can be discovered from the integrations for emission and absorption on a relatively course grid. Grid refinement is then used only in the regions of the peaks, thereby minimizing the number of evaluations of $\alpha$ and $j$ along the ray. 
In the situation of significant transparency along the ray, the code approximates emission from multiple passes across the tokamak using the single-pass results and assuming wall reflection with a given coefficient. Mode conversion at the wall is not considered. This simplification is made in view of the very complicated, machinespecific nature of the vacuum vessel. Resulting total radiation $I_{0}$ is given by ${ }^{8}$

$$
I_{o t}=I_{0} \sum_{k=0}^{\infty} r^{k} \exp (-k \tau)=I_{0} /(1-r \exp (-\tau)),
$$

where $I_{0}$ and $\tau$ are the radiation and optical depth resulting from one single pass across the tokamak and $r$ is the reflection coefficient.

\section{II.C. Optimal OXB Launch and the Mode Conversion Procedure}

O-mode radiation can penetrate into an overdense plasma, $X_{e} \equiv\left(\omega_{p e} / \omega\right)^{2}>1$, with $100 \%$ efficiency if it is incident in a plane plasma perpendicular to the flux surface and at an optimal angle $n_{\|, \text {opt }}= \pm \sqrt{Y_{e} /\left(1+Y_{e}\right)}$ with respect to the equilibrium magnetic field, ${ }^{10,11}$ where $Y_{e} \equiv$ $\omega_{c e} / \omega$. In this case $\mathrm{O}$-mode converts to $\mathrm{X}$-mode at the $X_{e}=1$ layer and continues to propagate into the plasma, subsequently converting to Bernstein mode. For a given antenna location, an iterative procedure is used to determine the launch angles giving $n_{\|, o p t}$ at the plasma frequency layer. To simulate a finite aperture to the receiving antenna, rays are also considered at nonoptimal angles. For such a ray, the power is decreased at mode conversion in accord with the Mjölhus transmission coefficient ${ }^{11}$ :

$$
\begin{aligned}
T\left(\hat{n}_{\perp}, n_{\|}\right)= & \exp \left\{-\frac{\pi \omega L_{n}}{c}\right\} \\
& \times \sqrt{\frac{Y}{2}}\left[2(1+Y)\left(n_{\|, o p t}-n_{\|}\right)^{2}+\hat{n}_{\perp}^{2}\right],
\end{aligned}
$$

where

$$
\begin{aligned}
\hat{n}_{\perp}= & \text { refractive index component lying in the flux } \\
& \text { surface and perpendicular to the magnetic field } \\
L_{n}= & \text { local density scale length } \\
\hat{n}_{\perp}= & \boldsymbol{n} \cdot(b \times \nabla \psi) /|(\boldsymbol{b} \times \nabla \psi)| .
\end{aligned}
$$

The unit vector $\boldsymbol{b}$ is along the ambient magnetic field, and $\psi$ is the poloidal magnetic flux function.

In order to obtain the BXO ray path for emission calculations, we launch $\mathrm{O}$-mode rays from the antenna. The ray penetrates into the plasma up to the O-mode cutoff at the magnetic surface $X_{e}\left(\rho_{\text {cutoff }}\right)=1$ and converts into the $X$-mode just inside the cutoff surface with $\rho<\rho_{\text {cutoff }}$, where $\rho_{\text {cutoff }}$ is the normalized minor radius of the cutoff. For OX mode conversion simulation at other than exactly the optimal angle, the O-mode ray at a point close to the cutoff surface, $X_{e r}(\rho)<1-\epsilon$, where $\epsilon$ is a small number, undergoes a small jump of the trajectory in the minor radius direction to the $\mathrm{X}$-mode cutoff point with $X_{e}(\rho)>1$. The $\mathrm{X}$-mode is launched from this point. At the shifted ray point, the $X$-mode wave power will be calculated as a product of the $\mathrm{O}$-mode wave power before the mode conversion multiplied by the transmission coefficient. O-mode rays from the antenna that do not approach closely enough to the $X_{e}\left(\rho_{\text {cutoff }}\right)=1$ surface, that is, do not approximately achieve $X_{e}\left(\rho_{\text {cutoff }}\right)=$ 1 , are reflected, and no power is transmitted by the OX conversion process. The poloidal projection of the ray near the OXB mode conversion region for NSTX emission is shown in Fig. 1 for a range of frequencies. The iteration procedure for $n_{\|, \text {opt }}$ gives the result that rays launched outside the plasma at $R=155 \mathrm{~cm}$ and $Z=$ $-5 \mathrm{~cm}$, intercept the plasma at $-7.07 \mathrm{~cm}$. Starting from outside the plasma, the rays show the small radial jump near the O-mode cutoff, the continuation into the plasma in the X-mode, group velocity reversal, ${ }^{15,16}$ returning to the UHR layer, linear conversion into the EBW, and continued propagation toward the central plasma. Group velocity reversal occurs very close to the plasma layer, at $\rho$, where $X_{e}=1+Y_{e}^{2}\left(1-n_{\|}^{2}\right)^{2} /\left(4 n_{\|}^{2}\right)$ (Refs. 15 and 16). These trajectories are used for the solution of the radiation transport equation back toward the antenna, giving the radiation collected by the antenna.

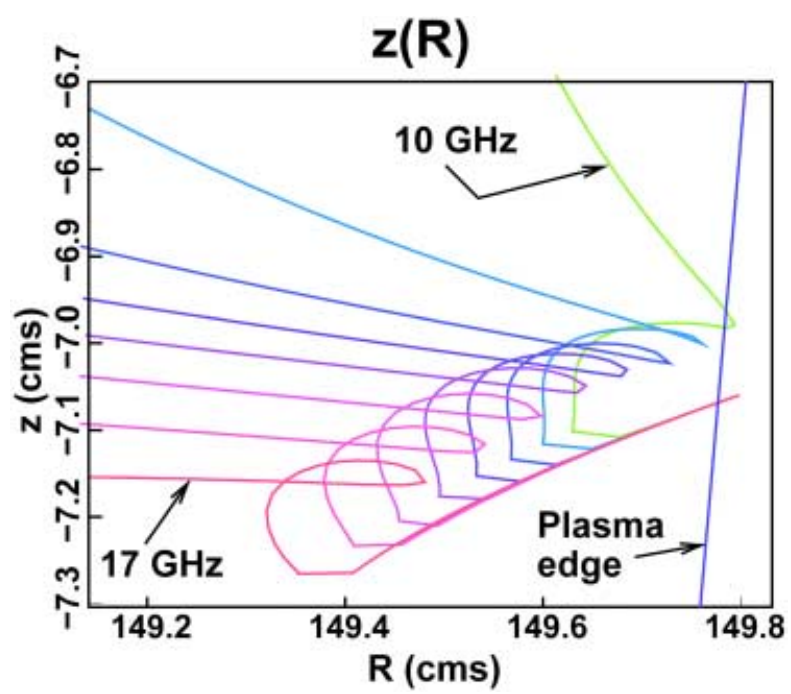

Fig. 1. Poloidal projection of the ray trajectories near NSTX OXB region. The results are for equispaced frequencies between the first and second edge cyclotron harmonic, 10 to $17 \mathrm{GHz}$. The near vertical line at the right is the plasma edge, with plasma density rapidly diminishing outside the last closed flux surface. Parallel reflective index $n_{\|}$at the plasma layer is near the optimum value, 0.6 . 


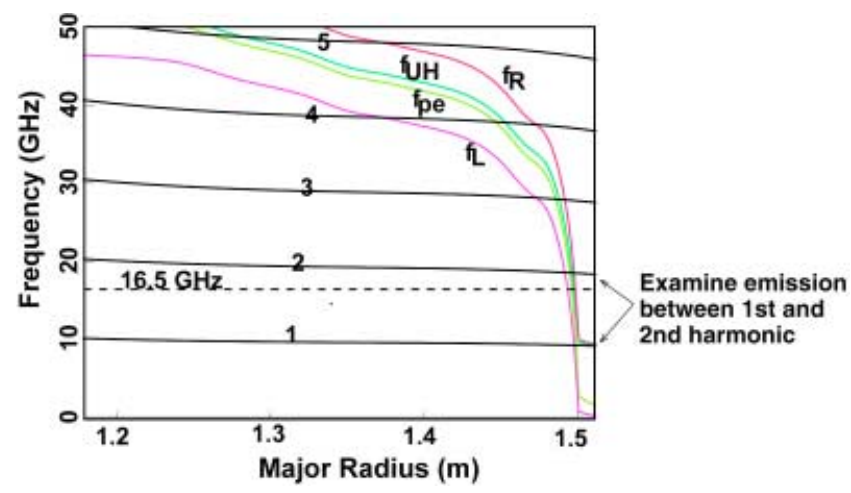

Fig. 2. Critical frequencies for the low-beta case. Curves are labeled by left-hand cutoff $f_{L}$, plasma $f_{\text {pe }}$, upper hybrid $f_{U H}$, and right-hand cutoff $f_{R}$ frequencies, and integers 1 through 5 are electron cyclotron harmonics.

\section{LOW-BETA EQUILIBRIUM RESULTS}

Figure 2 shows the critical frequencies for EBW in a low-beta $(\sim 10 \%)$ NSTX equilibrium for experimental shot 113544, examined in Ref. 2, as in the Fig. 1. Frequencies $f_{L}$ and $f_{R}$ are the $\mathrm{X}$-mode cyclotron cutoff frequencies for the left- and right-hand bounding surfaces for $n_{\|}=0$. For the more general case, $f_{L}, f_{R}$ are, respectively, the \pm expressions, $f_{L, R}=$ $1 / \sqrt{\left(1-n_{\|}^{2}\right)\left(1 \pm f / f_{c e}\right)}$. At $n_{\|}=n_{\|, o p t}$, frequency $f_{L}$ is reduced to the plasma frequency, enabling the $\mathrm{O}$ - and $\mathrm{X}$-mode cutoffs to be degenerated ${ }^{16}$ and giving $100 \%$ mode conversion. For the regime of frequencies near $16.5 \mathrm{GHz}$ that we study, this occurs very close to the plasma edge. Shot 113544 has a flattop current of $800 \mathrm{kA}$ and a toroidal field of $0.41 \mathrm{~T}$ on axis, and the central density and electron temperature are $3.6 \times 10^{13} \mathrm{~cm}^{-3}$ and $1.5 \mathrm{keV}$, which is typical of those viewed with the NSTX EBW radiometer system.

The toroidal and poloidal magnetic fields are comparable at the outer midplane. Coupling of the radiation out of the plasma at $16.5 \mathrm{GHz}$ agreed with theory, within the experimental uncertainty. We will focus on a range of frequencies between the first and second harmonic, as indicated in Fig. 2. As discussed above, O-mode rays from the antenna optimally convert to the $\mathrm{X}$-mode at $X_{e}(\rho)=1$. We have chosen $16.5 \mathrm{GHz}$ for this criterion and launch the other frequencies at these angles. The loss of transmitted power at the neighboring frequencies remains small.

Of course, for calculation of radiation from the thermal plasma, the main questions relate to the location of the radiation source for the EBW energy. For the nonthermal distributions, there are additional questions as to the sensitivity of EBW to the typically high-energy electrons. We use target nonthermal distributions as shown in Fig. 3, which are from a simulation of EBW current drive under anticipated conditions in NSTX. Figure 3a shows the electron velocity distribution near the radius of peak EBW current drive calculated for self-consistent
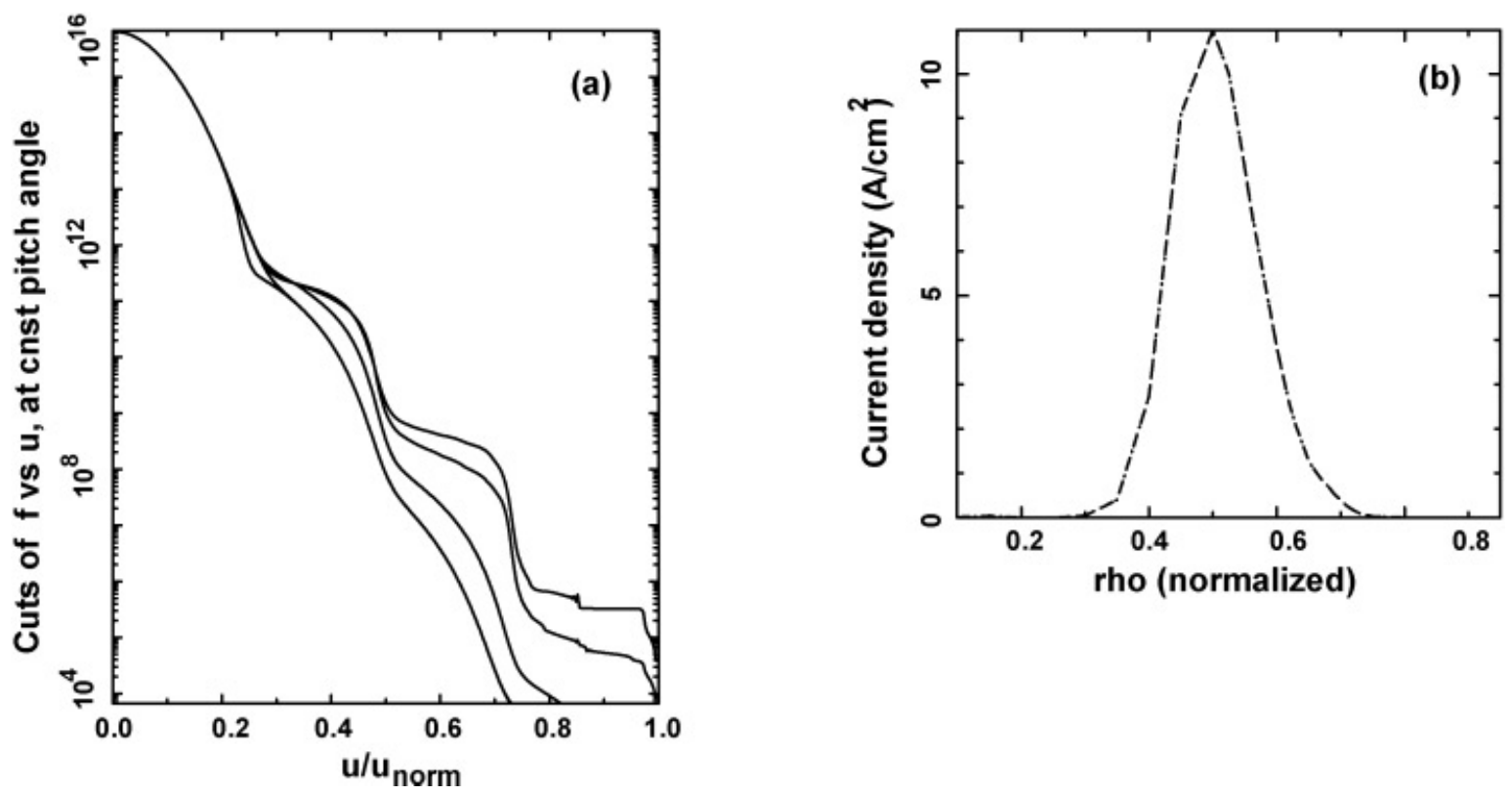

Fig. 3. (a) Cuts through the electron distribution near the radius corresponding to the maximum EBW driven current; $u_{\text {norm }}$ is such that maximum energy is $100 \mathrm{keV}$. (b) The driven current profile. 

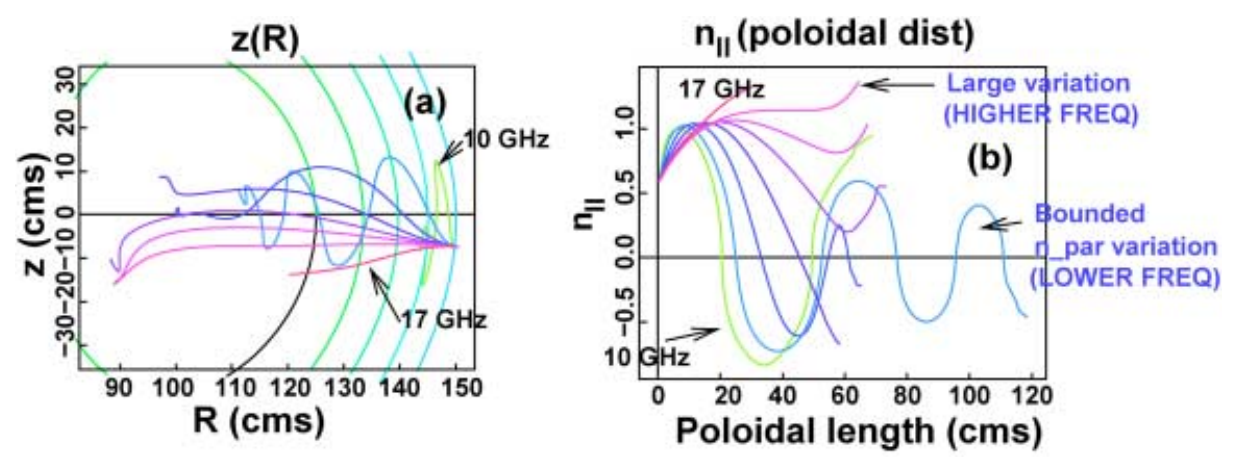

Fig. 4. (a) Ray trajectories and (b) n_parallel versus poloidal distance along the trajectory for an equispaced sequence of frequencies from 10 to $17 \mathrm{GHz}$.

EBW absorption and QL distortion with the CQL3D Fokker-Planck. ${ }^{14}$ The "staircase" effect in the tail of the distribution is due to finite-gyroradius effects on cyclotron damping as given by the $J_{1}^{2}\left(k_{\perp} v_{\perp} / \omega_{c e}\right)$ Bessel function variation of the quasi-linear diffusion coefficient. ${ }^{1}$ Figure $3 \mathrm{~b}$ shows the current profile obtained from distributions calculated on the plasma radial grid. The nonthermal electron tail peaks at $\rho=0.5$. These calculations are for OXB injection into plasma shot 113544 plasma at frequency $16.5 \mathrm{GHz}$. The EBW waves readily penetrate toward the plasma center and, because of $n_{\|}$ variation along the ray, are second-harmonic cyclotron absorbed.

Turning now to calculation of the EBW emission, Fig. 4 shows ray paths and the $n_{\|}$variation along the rays for a set of equispaced frequencies from 10 to $17 \mathrm{GHz}$, which is between the first and second harmonics as shown in Fig. 2. Transmission through the edge plasma is via the OXB mode conversion process, as shown clearly in Fig. 1. We distinguish two types of trajectory behavior. At the lower frequencies, just above the first harmonic, the wave trajectories and $n_{\|}$vary with distance in an oscillatory manner about the midplane of the plasma. As is typical of many EBW ray situations, for near equatorial plane launch, the $n_{\|}$variation is bounded. In contrast, at the higher frequencies but below the second harmonic, $n_{\|}$varies more secularly; $n_{\|}$is monotonically increasing with distance, as is typical of off-midplane launch.

The solution of the radiation transport equation for a low-frequency range, 10 to $12 \mathrm{GHz}$, and thermal plasma, then gives the results shown in Fig. 5a for the cumulative radiation intensity $I(R)$ flowing to the antenna, where $R$ is the plasma major radius. Each of these curves arises from radiation near the first harmonic. The most remarkable feature is the staircase effect in the radiation. This effect can be understood from Fig. 5b, which schematically shows (as elaborated in Ref. 17) the $v_{\|}$range of the relativistic resonance ellipse $\left(\left|n_{\|}\right|<1\right)$, accounting for the $1 / R$ variation of the equilibrium magnetic field. Thus, in terms of $\boldsymbol{u} \equiv \boldsymbol{p} /(m c)$, the relativistic resonance condition at the $n$ 'th harmonic can be written

$$
\left(u_{\|}-u_{\| 0}\right)^{2}+u_{\perp}^{2} /\left(1-n_{\|}^{2}\right)=u_{0}^{2},
$$

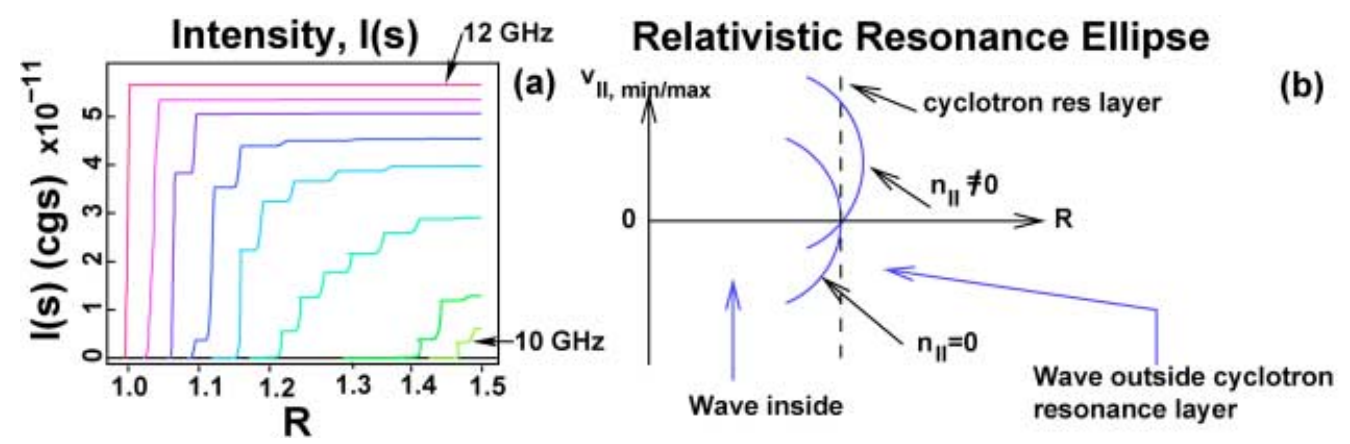

Fig. 5. (a) Cumulative emission intensity directed toward the antenna for frequencies in the range 10 to $12 \mathrm{GHz}$ (b) Parallel velocity range of the resonance ellipse versus major radius $R$. 
High Frequency, $z=-5 \mathrm{cms}$

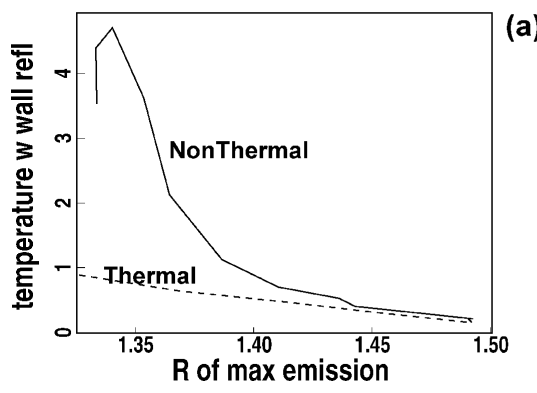

Low Frequency, $z=-5 \mathrm{cms}$

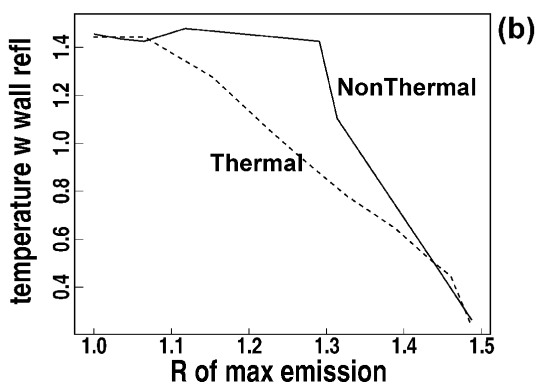

Low Frequency, $\mathrm{Z}=+15 \mathrm{cms}$

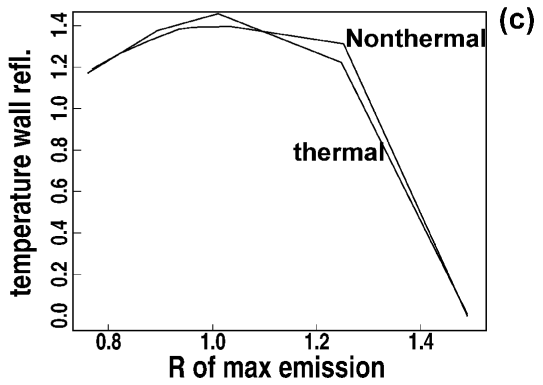

Fig. 6. Radiation temperature versus major radius $R$ of maximum emission for (a) low frequency, $z=-5 \mathrm{~cm}$; (b) high frequency, $z=-5 \mathrm{~cm}$; and (c) low frequency, $z=+15 \mathrm{~cm}$.

where

$$
u_{\| 0}=\left(n \omega_{c e}\right) n_{\|} /\left(1-n_{\|}^{2}\right)
$$

and

$$
u_{0}^{2}=\left[n \omega_{c e} / \omega-\left(1-n_{\|}^{2}\right)\right] /\left(1-n_{\|}^{2}\right)^{2} .
$$

In Fig. 5b, we plot the $u_{\|}$limits of the resonance ellipse as a function of major radius $R$. For $n_{\|}=0$, only electrons in the range $\left[v_{\| \min }, v_{\| \max }\right]$ and at major radius inside the cyclotron radius (the vertical dashed line) can resonate with the wave. Moreover, the resonance ellipse becomes an unshifted circle for $n_{\|}=0$. For $n \neq 0$, the Doppler-shifted resonance with electrons outboard of the cyclotron layer becomes possible. Thus, the staircase effect in Fig. 5a can be understood as due to the oscillation of $n_{\|}$about zero, as shown for the low frequencies in Fig. 4b. As ray energy at each frequency propagates toward its cyclotron layer, and $n_{\|}$oscillates, damping occurs when $\left|n_{\|}\right|$becomes sufficiently large that the radial Doppler shift reaches to the ray position, and indeed there is a one-to-one correspondence between peaks of $\left|n_{\|}\right|$and the steplike increases in $I(R)$ in Fig. 5a. From the above resonance ellipse equation, the largest outward shift in major radius occurs when $u_{0}=0$, that is, when $R / R_{\text {res }}=\left(1-n_{\|}^{2}\right)^{-1} \leq 1.3$, where $R_{\text {res }}$ is the radius at which cyclotron resonance occurs, of order the major radius; we have used $n_{\|} \leq 0.5$ per Fig. $4 \mathrm{~b}$, and have approximated the magnetic field variation as $1 / R$. Thus, the Doppler shift reaches $\sim 0.65 a$ outside the resonant layer, where $a$ is the minor radius. We expect this damping to be primarily on lower-velocity electrons. The radiation temperature calculated with the nonthermal distributions, as in Fig. 3, would therefore still largely represent the thermal electrons.

For the high-frequency radiation along rays in the frequency range 16 to $18 \mathrm{GHz}$, the $n_{\|}$variation is secular and larger than for the low frequencies. There will be a larger Doppler shift, and we expect a greater sensitivity to nonthermal electrons. This is borne out by the results shown in Fig. 6. The low-frequency emission to the an- tenna shown in Fig. 6a is not greatly enhanced from that obtained in the thermal case. On the other hand, the highfrequency range shown in Fig. 6b exhibits greatly enhanced nonthermal radiation.

We have also cursorily examined the effect of varying the poloidal angle of the antenna. Locating the antenna $15 \mathrm{~cm}$ above the midplane (and $R=155 \mathrm{~cm}$ ) gives radiation temperatures for the low-frequency range as shown in Fig. 6c. The $n_{\|}$variation is more localized near zero than in the previous results [which were for the antenna at $5 \mathrm{~cm}$ below the midplane (and $R=155 \mathrm{~cm}$ )] resulting in a more thermal temperature measurement, even for the nonthermal case. However, for an antenna location near the top of the plasma (or bottom), we had to use the higher-frequency range, and the $n_{\|}$variation was very pronounced and monotonic. The emission came from the outer $10 \mathrm{~cm}$ of the plasma.

\section{HIGH-BETA EQUILIBRIUM RESULTS}

Results in this section are for a high-beta $(\sim 41 \%)$ model equilibrium previously examined in Ref. 3 . This shot has a current of 1.0 MA, a toroidal field of $0.30 \mathrm{~T}$ on axis, and central plasma density/temperature equal to $3 \times 10^{13} \mathrm{~cm}^{-3} / 2.0 \mathrm{keV}$. The poloidal field is slightly greater than the toroidal field at the outboard edge of the plasma. Figure 7 shows the critical frequencies versus major radius. The variation of the gyrofrequency indicates that a magnetic well is present. This further restricts ray penetration to the plasma center. We have examined a range of frequencies, from 8.4 to $25.1 \mathrm{GHz}$, covering the first to the third central cyclotron frequency harmonics. Some rays with moderate $n_{\|}$variation can penetrate to near the plasma center before being substantially damped in the thermal plasma case and therefore emit from there.

Effects of a nonthermal plasma, as studied in Ref. 3, are also considered, giving in this particular case nonthermal distributions localized to $\rho \sim 0.7$. For an input 


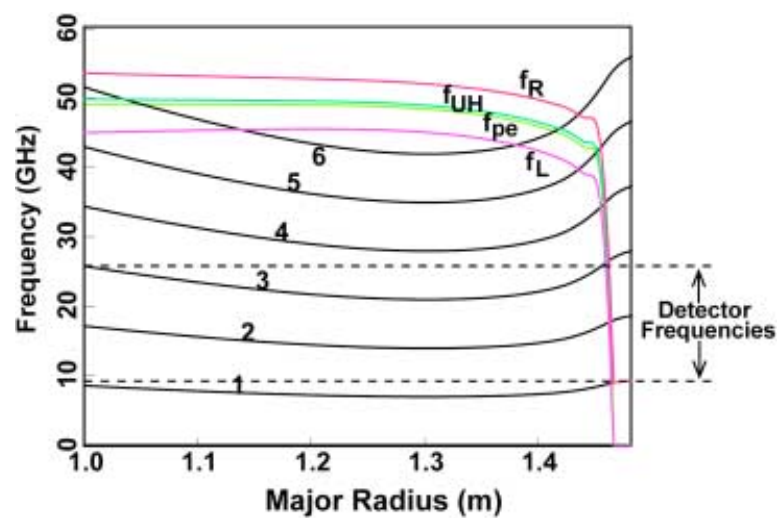

Fig. 7. Critical frequencies versus major radius $R$ for a highbeta equilibrium. Labels as in Fig. 2.

power of $4 \mathrm{MW}$, these distributions otherwise appear similar to those in Fig. 3.

Figure 8 shows radiation temperature versus $f / f_{c e} 0$ for (a) the thermal plasma and (b) the nonthermal plasma. Frequency $f_{c e 0}$ is cyclotron frequency evaluated at the magnetic axis. For frequencies above the second harmonic, emission comes from radii up to near the plasma center as seen in Fig. 8a where maximum radiation temperature approaches $2 \mathrm{keV}$, the central temperature of the Maxwellian target. For the nonthermal target, the high

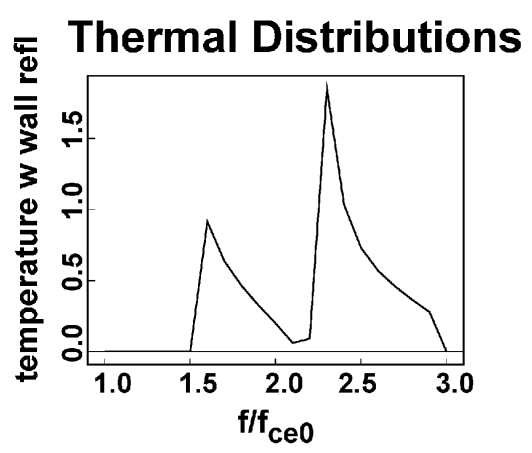

(a)

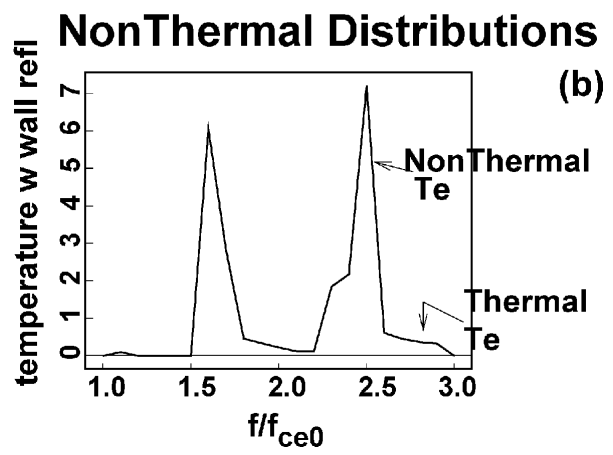

Fig. 8. Radiation temperature versus $f / f_{c e 0}$ derived from (a) thermal and (b) nonthermal distributions. High-beta case. radiation temperatures in Fig. $8 \mathrm{~b}$ show that the nonthermal electrons dominate the radiation from the radial region $\rho \sim 0.7$ where they appear, into near the plasma center.

\section{CONCLUSIONS}

The GENRAY ray-tracing code has been augmented to solve the radiation transport equation, and application has been made for EBWE observed at the plasma edge from both thermal and general nonthermal distribution functions. The specific equations solved and aspects of the numerical methods have been given. This analysis is pertinent to future EBW heating and current drive experiments such as on NSTX.

It is found that the EBWE provides a flexible measurement of thermal and nonthermal electron distributions over the range of plasma beta attained in spherical tokamak experiments. At low beta, central plasma thermal and nonthermal radiation temperature measurements are possible. At high beta, central thermal measurements are more difficult and may not be possible if there are intervening nonthermal distributions. Full interpretation of the measurements will involve careful modeling of the electrons, and in some cases the data will only indicate consistency with the model. That is, determination of the sensitivity of EBWE to tail electrons necessitates an interplay between the experimental measurements and the modeling. These studies can also provide a consistency check that the physics of the experiment is well understood.

\section{ACKNOWLEDGMENT}

This research is supported by U.S. Department of Energy contract DE-FG03-02ER54684.

\section{REFERENCES}

1. T. H. STIX, Waves in Plasmas, American Institute of Physics, New York (1992).

2. G. TAYLOR et al., Phys. Plasmas, 12, 052511 (2005).

3. G. TAYLOR et al., Phys. Plasmas, 11, 4733 (2004).

4. V. SHEVCHENKO, Y. BARANOV, M. O'BRIEN, and A. SAVELIEV, Phys. Rev. Lett., 89, 265005 (2002).

5. C. B. FOREST, P. K. CHATTOPADHAY, R. W. HARVEY, and A. P. SMIRNOV, Phys. Plasmas, 7, 1352 (2000).

6. A. P. SMIRNOV and R. W. HARVEY, Bull. Am. Phys. Soc., 40, 1837, abstract 8p35 (1995).

7. G. BEKEFI, Radiation Processes in Plasmas, John Wiley and Sons, New York (1966).

8. R. W. HARVEY, M. R. O'BRIEN, M. G. McCOY, and G. D. KERBEL, in Proc. 7th Joint Workshop and International Atomic 
Energy Agency Technical Committee Meeting on Electron Cyclotron Emission and Electron Cyclotron Resonance Heating, Hefei, China, 1989; see also R. W. HARVEY, M. R. O'BRIEN, V. ROZHDESTVESKY, T. C. LUCE, M. G. McCOY, and G. D. KERBEL, Phys. of Fluids B, 5, 446 (1993).

9. A. K. RAM and S. D. SCHUlTZ, Phys. Plasmas, 7, 4084 (2000).

10. V. KOPECKY, J. PREINHAELTER, and J. VACLAVIK, J. Plasma Phys., 3, 179 (1969).

11. E. MJÖLHUS, J. Plasma Phys., 31, 7 (1984).

12. H. P. LAQUA et al., Phys. Rev. Lett., 78, 18 (1997).
13. M. BORNATICI, R. CANO, O. DeBARBIERI, and F. ENGELMANN, Nucl. Fusion, 23, 1153 (1983).

14. R. W. HARVEY and M. G. McCOY, "The CQL3D FokkerPlank Code," International Atomic Energy Agency Technical Committee Meeting, Montreal, 1992; available as USDOC, NTIS document DE93002926.

15. H. WEITZNER and D. B. BATCHELOR, Phys. Fluids, 22, 1355 (1979).

16. R. A. CAIRNS and C. N. LASHMORE-DAVIES, Phys. Plasmas, 7, 4126 (2000).

17. R. W. HARVEY, M. G. McCOY, and G. D. KERBEL, Phys. Rev. Lett., 62, 426 (1989). 